\title{
Robust High Throughput Space Time Block Codes Using Parallel Interference Cancellation
}

\author{
Nicholas S. J. Pau, Desmond P. Taylor, Life Fellow, IEEE, and Philippa A. Martin, Senior Member, IEEE
}

\begin{abstract}
We present a space-time coded system which achieves high throughput and good performance using lowcomplexity detection and decoding. We focus on a Rate 2 quasiorthogonal space-time block code structure which enables us to achieve an overall throughput of $5.6 \mathrm{bits} / \mathrm{symbol}$ period with good performance and relatively simple decoding using iterative parallel interference cancellation. We show that this can be achieved through the use of a bit-mapped coded modulation (BMCM) structure using parallel short low density parity check codes. The proposed system is shown to perform well on flat Rayleigh fading channels with a wide range of normalized fade rates, and to be robust to channel estimation errors. A comparison with bit-interleaved coded modulation (BICM) is also provided.
\end{abstract}

Index Terms - Error correcting coding, iterative methods, low density parity check codes, MIMO systems, space-time coding.

\section{INTRODUCTION}

$\mathbf{T}$ HE goal of employing multiple transmit and receive antennas is to increase available channel capacity and improve performance over that of single antenna systems. In this paper, we design a space-time coded modulation scheme that (a) achieves high throughput, (b) provides good performance, (c) generates low processing delay and (d) uses a low complexity detection/decoding scheme. Most previous work [1], [2], [3], [4] on multiple-input multiple-output (MIMO) systems meets only two or three of these four criteria.

Direct transmission of coded modulation schemes [1] in MIMO systems achieves high throughput, at the expense of high detection complexity. The Bell-Labs Layered Space-Time (BLAST) schemes [2] achieve high throughput at comparatively lower complexity but have relatively poor performance. The concatenation of coded modulation schemes with orthogonal space-time block codes (OSTBCs) [3], [4] allows linear separation of the transmitted symbols at the receiver, prior to decoding. However, OSTBCs [5], [6] cannot offer rates greater than 1 symbol per time slot. To increase this, we consider quasi-orthogonal space-time block codes (QOSTBCs) [7], [8]. We focus on the double space time transmit diversity (DSTTD) scheme of [8], which is a Rate 2 QOSTBC that combines the Alamouti OSTBC [5] with BLAST [2]. Similar hybrid schemes can be found in [9], and [10] for space-time trellis codes.

Manuscript received June 6, 2006; revised November 12, 2006 and June 7, 2007; accepted September 13, 2007. The associate editor coordinating the review of this paper and approving it for publication was A. Grant.

The authors are with the Electrical and Computer Engineering Department, University of Canterbury, Private Bag 4800, Christchurch, New Zealand (email: \{nsp14, taylor, p.martin\}@elec.canterbury.ac.nz).

Digital Object Identifier 10.1109/TWC.2008.060335.
At the transmitter, the QOSTBC of [8] is serially concatenated with bit-mapped coded modulation (BMCM). This BMCM-QOSTBC structure uses parallel forward error correction (FEC) component codes, which allows parallel decoding in the receiver. Unlike bit-interleaved coded modulation (BICM) [11], BMCM does not require interleaving ${ }^{1}$ and so has shorter processing delays. We use parallel low density paritycheck (LDPC) component codes in the BMCM structure. This allows belief propagation (BP) decoding, which has modest decoding complexity, to be performed in parallel for each component code. This is attractive in practical applications because it reduces the overall processing delays.

OSTBCs can eliminate co-channel interference in multiple antenna transmissions using simple linear processing [5], [6]. However, following linear processing, some co-channel interference remains with QOSTBCs and this degrades performance. For the Rate 1 QOSTBC of [7], performance is improved by employing joint detection (JD), which has high detection complexity. For the Rate 2 QOSTBC of [8], ordered successive interference cancellation (SIC) [2], [10], [9] is used. Here, we employ iterative parallel interference cancellation (PIC) [12], [13], [14] to mitigate the co-channel interference. PIC is widely used in multi-user environments and is considered less complex than SIC [12]. As in [13], [14], iterations will be performed between the PIC detector and parallel FEC decoders. Diagonal-BLAST and convolutional codes are used in [14]. A performance comparison of layered space time codes is carried out in [13], where an improved version of the PIC detector uses decision statistics combining [15]. Here, we use a standard form of PIC to maintain simplicity. The systems in [13], [14] use PIC with BLAST schemes. Here, we employ PIC with QOSTBCs.

To date, most research on STBCs assumes quasi-static fading [5], [7], i.e. the channel stays constant for the duration of the STBC block and varies independently from block to block. Essentially this is a fast-fading channel. We employ this initially to allow a fair comparison with existing systems. We then investigate the performance of the BMCM-QOSTBC scheme in time-varying flat Rayleigh fading, over a wide range of normalized fade rates, and demonstrate its robustness to channel estimation errors.

In Section II, we describe the proposed BMCM-QOSTBC structure and the channel model. We define a power correlation coefficient to model channel estimation errors. Section III describes the different detection and decoding schemes used. Simulation results are presented in Section IV and compar-

\footnotetext{
${ }^{1}$ Our simulations have shown that using interleavers with BMCM in a fastfading channel provides no performance gain.
} 


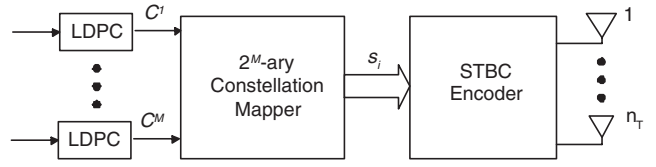

(a) Transmitter : BMCM-STBC structure

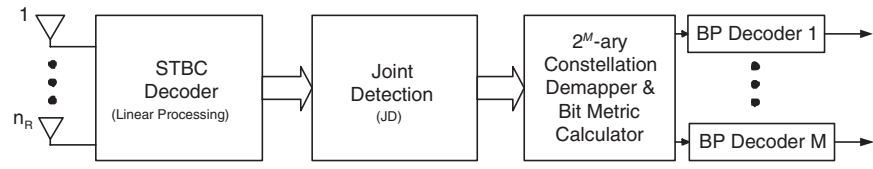

(b) Receiver : Joint Detection

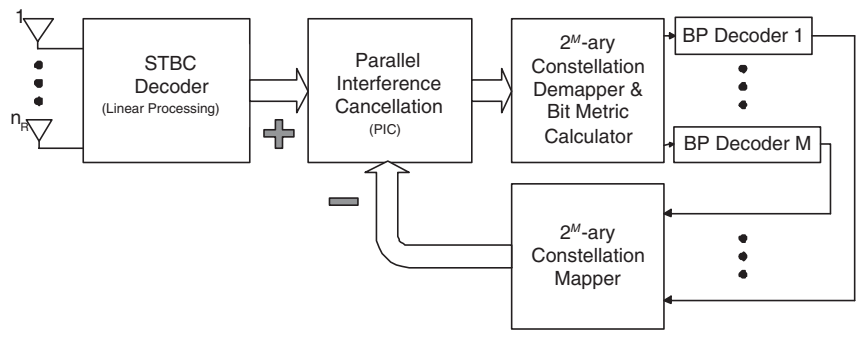

(c) Receiver : Parallel Interference Cancellation

Fig. 1. Transmitter and receiver structures.

isons to other systems are shown. For higher order constellations, we show how performance can be optimized. We show that the proposed system is robust to time-varying flat Rayleigh fading and to channel estimation errors. Conclusions are drawn in Section V.

\section{Proposed BMCM-QOSTBC System}

We consider a MIMO system with $n_{T} n_{R}$ independent subchannels between $n_{T}$ transmit and $n_{R}$ receive antennas. Initially, we assume quasi-static flat Rayleigh fading with ideal channel state information (CSI) available at the receiver, but not at the transmitter. We consider the effects of imperfect CSI in Section IV-B. In Section IV-D, we investigate the effects of allowing the fading to vary continuously according to a normalized fade rate.

\section{A. Encoding}

The proposed BMCM-QOSTBC structure is shown in Fig. 1a. We consider a $2^{M}$-ary constellation. The input data stream is demultiplexed into $M$ data substreams $\left\{\boldsymbol{B}^{m}\right\}_{m=1}^{M}$ with block lengths $K_{m}$. Each substream is encoded using an LDPC code $^{2}$ to obtain $M$ length- $N$ codewords $\left\{\boldsymbol{C}^{m}\right\}_{m=1}^{M}$, where the $i^{t h}$ encoded bit in $C^{m}$ is denoted $C_{i}^{m}$. Note the flexibility of the BMCM structure, where substreams can be encoded using LDPC codes with different values of $K_{m}$ as long as all $M$ codes produce codewords of the same length, $N$. The overall rate of the $M$ LDPC codes is then

$$
R_{l d p c}=\frac{\sum_{m=1}^{M} K_{m}}{M N} .
$$

The encoding process can be written as $\boldsymbol{C}^{m}=\boldsymbol{B}^{m} \boldsymbol{G}^{m}$, where $\boldsymbol{G}^{m}$ is the $K_{m}$-by-N generator matrix of the $m^{t h}$

\footnotetext{
${ }^{2}$ Any FEC codes may be used, but LDPC codes were chosen to keep decoding complexity low.
}

LDPC component code. The LDPC code construction is described in Appendix I. It novel to the best of our knowledge, and allows for a flexible choice of rates. The $i^{t h}$ bits from all $M$ LDPC codewords collectively select the $i^{\text {th }} 2^{M}$-ary constellation point $\boldsymbol{s}_{i}$.

The rate 1 QOSTBC in [7] is given by

$$
\zeta_{44}=\left[\begin{array}{cccc}
s_{1} & s_{2} & s_{3} & s_{4} \\
-s_{2}^{*} & s_{1}^{*} & -s_{4}^{*} & s_{3}^{*} \\
-s_{3}^{*} & -s_{4}^{*} & s_{1}^{*} & s_{2}^{*} \\
s_{4} & -s_{3} & -s_{2} & s_{1}
\end{array}\right]
$$

where each column is transmitted from a different antenna and each row is transmitted in a different time slot. This QOSTBC transmits four new constellation points $\left(s_{1}, s_{2}, s_{3}, s_{4}\right)$ using $n_{T}=4$ and $L=4$ time slots. The STBC rate is defined as

$$
R_{s t b c}=\frac{\text { number of new symbols transmitted, } w}{\text { number of time slots used, } L} .
$$

Here we consider the DSTTD scheme of [8] which can be described by

$$
\zeta_{42}=\left[\begin{array}{cccc}
s_{1} & s_{2} & s_{3} & s_{4} \\
-s_{2}^{*} & s_{1} & -s_{4}^{*} & s_{3}
\end{array}\right],
$$

where four new constellation points are transmitted using $n_{T}=4$ and $L=2$ time slots. This is a truncated version of the Rate 1 QOSTBC in (2). If we split the transmit antennas into Group A (Tx1, Tx2) and Group B (Tx3, Tx4), we are effectively transmitting the two groups independently (as in BLAST schemes), where each group uses the simple Alamouti OSTBC. Therefore, DSTTD is quasi-orthogonal and has $R_{s t b c}=2$. We shall refer to it as the Rate 2 QOSTBC. The throughput for a system using a $2^{M}$-ary constellation is then defined as

$$
\text { Throughput }=M R_{s t b c} R_{l d p c} \quad \text { bits/symbol period. }
$$

Compared to the more well-known BICM structure [11], BMCM allows parallel processing, which suits implementation using the increasingly popular field-programmable gate arrays (FPGAs) [16]. For the same frame length (containing approximately the same number of information bits), the BMCM structure uses $M$ components codes which are $M$ times shorter than the single code in the BICM structure, for $2^{M}$-ary constellations. Therefore, the processing delay required for $\mathrm{BMCM}$ is only $\frac{1}{M}$ that of BICM. In addition, BICM suffers from further processing delays due to the use of interleavers and deinterleavers.

\section{B. Channel Model}

We consider Rayleigh flat fading and let $\alpha_{p}^{q}(t)$ denote the complex fading coefficient affecting the symbol $x_{p}(t)$ at time $t$ for the subchannel between the $p^{t h}$ transmit antenna and the $q^{t h}$ receive antenna for $p=1,2, \ldots, n_{T}$ and $q=$ $1,2, \ldots, n_{R}$. We model the coefficients as zero-mean complex Gaussian random variables with variance $1 / 2$ per dimension. We consider three time-varying channel models (from less to more practical):

QS-Ind channel: This is the most common channel model in the literature [5], [7]. The fading coefficients remain fixed during each $\mathrm{STBC}$ block of $L$ time slots 
and vary independently from one block to the next (quasi-static fading).

QS-fDT channel: The fading coefficients remain fixed during each STBC block but vary according to the normalized $f_{D} T$ from one block to the next ${ }^{3}$, where the maximum Doppler shift $f_{D}$ is normalized to the symbol period $T$.

NoQS-fDT channel: The quasi-static fading assumption is removed. The fading coefficients vary continuously from one time slot to the next according to $f_{D} T$.

CSI is assumed to be available at the receiver, but not at the transmitter. To consider the effect of imperfect CSI, we model the estimated $\mathrm{CSI}^{4}$ coefficients as

$$
\hat{\alpha}=\rho \alpha+\sqrt{1-\rho^{2}} \epsilon, \quad 0 \leq \rho \leq 1
$$

where $\alpha$ is the actual CSI, $\rho$ is the power correlation coefficient between $\alpha^{2}$ and $\hat{\alpha}^{2}$, and $\epsilon$ is an independent zero-mean complex Gaussian random variable with variance $1 / 2$ per dimension. This model is used so that the estimated CSI has the same variance as the actual CSI. We specify the estimation noise energy as $\frac{1-\rho^{2}}{\rho^{2}} \times 100 \%$ with respect to the actual CSI energy. When $\rho=1$, we have $\hat{\alpha}=\alpha$, meaning perfect CSI is available.

We fix the total transmitted energy across all $n_{T}$ transmit antennas to be 1 for each time slot. For $n_{T}=4$, the symbol transmitted from each antenna then contains $\frac{1}{n_{T}}=1 / 4$ unit of energy. The sampled signal at the $q^{t h}$ receive antenna is a noisy superposition of the faded transmitted signals given by

$$
r^{q}(t)=\sum_{p=1}^{n_{T}} \alpha_{p}^{q}(t) x_{p}(t)+w^{q}(t), \quad t=1, \ldots, L,
$$

where $w^{q}(t)$ is the additive white Gaussian noise (AWGN) at the $q^{\text {th }}$ receive antenna at time $t$ for $q=1,2, \ldots, n_{R}$. It is modeled as an independent complex Gaussian random variable with zero mean and a one-dimensional noise variance defined by

$$
\sigma^{2}=\frac{N_{0}}{2}=\frac{n_{T} E_{s}}{M R_{s t b c} R_{l d p c} 10^{0.1 S N R}},
$$

where $E_{s}$ is the average energy of a constellation point and $S N R$ is the signal-to-noise ratio per receive antenna in decibels $(d B)$.

\section{Detection And Decoding}

At the receiver, linear processing [5], [6] is first applied to the received signals to produce soft estimates of the transmitted symbols. Assuming perfect CSI, this produces estimates of the transmitted symbols $s_{1}, s_{2}, s_{3}, s_{4}$, as in (9) and (10) for the Rate 1 and Rate 2 QOSTBCs, respectively. These equations assume quasi-static fading and therefore the time notation is dropped from the $\alpha_{p}^{q}$ terms since $\alpha_{p}^{q}(1)=\alpha_{p}^{q}(2)=$ $\ldots=\alpha_{p}^{q}(L)$. When this assumption is removed (e.g. NoQSfDT channel), the $\alpha_{p}^{q}$ terms vary according to $f_{D} T$. However, linear processing is still performed using the estimate $\hat{\alpha}_{p}^{q}(1)$

\footnotetext{
${ }^{3}$ Using a third-order FIR filter, we generate fading coefficients which vary according to $f_{D} T$, but we only use every $L^{t h}$ coefficient.

${ }^{4}$ Actual estimation of CSI at the receiver is beyond the scope of the present paper. Only modelling of a random estimation error is considered.
}

for all $L$ time slots, as if the fading were quasi-static. This maintains the simplicity of the linear processing.

$$
\begin{aligned}
\hat{\boldsymbol{s}}_{\mathbf{1}}= & \sum_{q=1}^{n_{R}}\left(\left|\alpha_{1}^{q}\right|^{2}+\left|\alpha_{2}^{q}\right|^{2}+\left|\alpha_{3}^{q}\right|^{2}+\left|\alpha_{4}^{q}\right|^{2}\right) \boldsymbol{s}_{\mathbf{1}} \\
& +\sum_{q=1}^{n_{R}} 2\left(\alpha_{1}^{q} \alpha_{4}^{q *}-\alpha_{2}^{q} \alpha_{3}^{q *}\right) s_{\mathbf{4}}+\text { noise } \\
\hat{\boldsymbol{s}}_{\mathbf{2}}= & \sum_{q=1}^{n_{R}}\left(\left|\alpha_{1}^{q}\right|^{2}+\left|\alpha_{2}^{q}\right|^{2}+\left|\alpha_{3}^{q}\right|^{2}+\left|\alpha_{4}^{q}\right|^{2}\right) \boldsymbol{s}_{\mathbf{2}} \\
& +\sum_{q=1}^{n_{R}} 2\left(\alpha_{2}^{q} \alpha_{3}^{q *}-\alpha_{1}^{q} \alpha_{4}^{q *}\right) \boldsymbol{s}_{\mathbf{3}}+\text { noise } \\
\hat{\boldsymbol{s}}_{\mathbf{3}}= & \sum_{q=1}^{n_{R}}\left(\left|\alpha_{1}^{q}\right|^{2}+\left|\alpha_{2}^{q}\right|^{2}+\left|\alpha_{3}^{q}\right|^{2}+\left|\alpha_{4}^{q}\right|^{2}\right) \boldsymbol{s}_{\mathbf{3}} \\
& +\sum_{\text {desired terms }}^{\sum_{q=1}^{n_{R}} 2\left(\alpha_{2}^{q} \alpha_{3}^{q *}-\alpha_{1}^{q} \alpha_{4}^{q *}\right) s_{\mathbf{2}}+\text { noise }} \\
\hat{\boldsymbol{s}}_{\mathbf{4}}= & \sum_{\text {interference terms }}^{\sum_{q=1}^{n_{R}}\left(\left|\alpha_{1}^{q}\right|^{2}+\left|\alpha_{2}^{q}\right|^{2}+\left|\alpha_{3}^{q}\right|^{2}+\left|\alpha_{4}^{q}\right|^{2}\right) \boldsymbol{s}_{\mathbf{4}}} \\
& +\underbrace{}_{\sum_{q=1}^{n_{R}} 2\left(\alpha_{1}^{q} \alpha_{4}^{q *}-\alpha_{2}^{q} \alpha_{3}^{q *}\right) \boldsymbol{s}_{\mathbf{1}}+\text { noise }}
\end{aligned}
$$

$$
\begin{aligned}
\hat{\boldsymbol{s}}_{\mathbf{1}}= & \sum_{q=1}^{n_{R}}\left(\left|\alpha_{1}^{q}\right|^{2}+\left|\alpha_{2}^{q}\right|^{2}\right) \boldsymbol{s}_{\mathbf{1}} \\
& +\sum_{q=1}^{n_{R}}\left(\alpha_{1}^{q *} \alpha_{3}^{q}+\alpha_{2}^{q} \alpha_{4}^{q *}\right) s_{\mathbf{3}}+\sum_{q=1}^{n_{R}}\left(\alpha_{1}^{q *} \alpha_{4}^{q}-\alpha_{2}^{q} \alpha_{3}^{q *}\right) s_{\mathbf{4}}+\text { noise } \\
\hat{\boldsymbol{s}}_{\mathbf{2}}= & \sum_{q=1}^{n_{R}}\left(\left|\alpha_{1}^{q}\right|^{2}+\left|\alpha_{2}^{q}\right|^{2}\right) \boldsymbol{s}_{\mathbf{2}} \\
& +\sum_{q=1}^{n_{R}}\left(\alpha_{2}^{q *} \alpha_{4}^{q}+\alpha_{1}^{q} \alpha_{3}^{q *}\right) \boldsymbol{s}_{\mathbf{4}}+\sum_{q=1}^{n_{R}}\left(\alpha_{2}^{q *} \alpha_{3}^{q}-\alpha_{1}^{q} \alpha_{4}^{q *}\right) \boldsymbol{s}_{\mathbf{3}}+\text { noise }
\end{aligned}
$$$$
\hat{s}_{\mathbf{3}}=\sum_{q=1}^{n_{R}}\left(\left|\alpha_{3}^{q}\right|^{2}+\left|\alpha_{4}^{q}\right|^{2}\right) s_{3}
$$$$
+\sum_{q=1}^{n_{R}}\left(\alpha_{3}^{q *} \alpha_{1}^{q}+\alpha_{4}^{q} \alpha_{2}^{q *}\right) s_{1}+\sum_{q=1}^{n_{R}}\left(\alpha_{3}^{q *} \alpha_{2}^{q}-\alpha_{4}^{q} \alpha_{1}^{q *}\right) s_{2}+\text { noise }
$$$$
\hat{s}_{4}=\underbrace{\sum_{q=1}^{n_{R}}\left(\left|\alpha_{3}^{q}\right|^{2}+\left|\alpha_{4}^{q}\right|^{2}\right) \boldsymbol{s}_{4}}_{\text {desired terms }}
$$

$$
+\underbrace{\sum_{q=1}^{n_{R}}\left(\alpha_{4}^{q *} \alpha_{2}^{q}+\alpha_{3}^{q} \alpha_{1}^{q *}\right) s_{2}+\sum_{q=1}^{n_{R}}\left(\alpha_{4}^{q *} \alpha_{1}^{q}-\alpha_{3}^{q} \alpha_{2}^{q *}\right) s_{1}}_{\text {interference terms }}+\text { noise. }
$$

The interference terms in (9) and (10) are due to the loss of orthogonality in the QOSTBC structures of (2) and (4). The soft symbol estimates from the linear processing are used by either the JD scheme of [7] or the proposed PIC scheme to produce better estimates, as explained below. 


\section{A. Joint Detection}

The receiver structure for the JD scheme of [7] is shown in Fig. 1b. The Rate 1 QOSTBC of [7] produces estimates that depend on the transmitted symbol plus one interferer and some noise terms, as shown in (9). The JD scheme considers all possible pairs of constellation points consisting of a transmitted symbol and an interferer, and selects the best pair based on Euclidean distance. The improved estimates are then transformed into the corresponding bit metrics $f_{m, i}^{0}$ and $f_{m, i}^{1}$ for the $M$ BP decoders, calculated using (11) and (15) in Section III-C.

As seen in (9), linear processing on the Rate 1 QOSTBC decouples the transmitted symbols into two independent pairs $\left(s_{1}, s_{4}\right.$ and $\left.s_{2}, s_{3}\right)$. This is a special case that allows a reduced search complexity of $2\left(2^{M}\right)^{2}$ using pairwise JD. The complexity increases as $\left(2^{M}\right)^{w}$ for higher rate QOSTBCs, where $w$ is the number of new symbols per QOSTBC block and is typically given by $n_{T}$. For a BMCM system using 16QAM and the Rate 2 QOSTBC, JD requires a search through $16^{4}=65536$ possible combinations of four constellation points.

\section{B. Parallel Interference Cancellation}

The exponentially increasing complexity of JD with $n_{T}$ motivates the use of a simpler detection scheme such as PIC. In [14], information is iteratively shared between a PIC block and $n_{T}$ convolutional decoders, via interleaving/de-interleaving. In [13], information is iteratively shared between the PIC block and $n_{T}$ LDPC decoders, again via interleaving/deinterleaving. Interleaving/de-interleaving generates additional processing delays. Here, information is iteratively exchanged between the PIC block and the $M$ parallel LDPC decoders, via mapping/demapping with no interleaving. The resulting receiver structure is shown in Fig. 1c.

In the first iteration, no PIC is performed following linear processing. The symbol estimates from the linear processing block are demodulated and the soft bit metrics $f_{m, i}^{0}$ and $f_{m, i}^{1}$ are passed directly to the $M$ parallel LDPC decoders. The hard bit decisions from the decoders are then re-modulated to give improved estimates of the transmitted symbols, which are then used with the CSI to perform soft interference cancellation according to (9) or (10). This process generates improved soft symbol estimates which are again demodulated and the corresponding soft bit metrics passed to the parallel LDPC decoders. This iterative process is repeated until there is negligible further improvement in performance.

The LDPC decoders use the BP decoding algorithm, which requires internal iterations. These differ from the iterations between the PIC block and the LDPC decoders described above. We use the terms BP iterations and PIC updates to distinguish the two iterative processes.

The detection complexity of PIC is only $w\left(2^{M}\right)$ per PIC update $^{5}$ for any QOSTBCs, since each transmitted symbol is individually detected. This represents a linear increase in complexity for PIC compared to a polynomial increase for $\mathrm{JD}$, as throughput is increased through constellation size.

\footnotetext{
${ }^{5}$ Typically, 4-9 PIC updates is sufficient to achieve good performance.
}

When $n_{T}$ increases, $w$ also increases, but PIC maintains a linear growth in detection complexity whereas JD suffers from exponential growth.

\section{Derivation of the Bit Metric to the BP Decoders.}

The outputs of the JD or PIC detection blocks are the soft symbol estimates, $\hat{\boldsymbol{s}}_{i}$, of (9) or (10). The $m^{\text {th }}$ BP decoder requires as input bit metrics $f_{m, i}^{0}=P\left(C_{i}^{m}=0 \mid \hat{\boldsymbol{s}}_{i}\right)$ and $f_{m, i}^{1}=P\left(C_{i}^{m}=1 \mid \hat{\boldsymbol{s}}_{i}\right)$. We calculate these bit metrics from the symbol estimates using

$$
f_{m, i}^{0}=\frac{1}{1+e^{\lambda_{i}^{m}}}, \quad f_{m, i}^{1}=\frac{1}{1+e^{-\lambda_{i}^{m}}}
$$

so that $f_{m, i}^{0}+f_{m, i}^{1}=1$, and we define

$$
\lambda_{i}^{m}=\ln \frac{P\left(C_{i}^{m}=1 \mid \hat{\boldsymbol{s}}_{i}\right)}{P\left(C_{i}^{m}=0 \mid \hat{\boldsymbol{s}}_{i}\right)} .
$$

The soft estimated symbol from the JD or PIC block can be written as $\hat{\boldsymbol{s}}_{i}=K \boldsymbol{s}_{i}+n_{i}$, where $K$ is the coefficient of $\boldsymbol{s}_{i}$ in (9) or (10), and $n_{i}$ is the sum of the interference and receiver noise terms. We assume that $n_{i}$ is a complex Gaussian random variable. Denote $\chi_{i}^{m,(0)}$ as the set of symbols associated with $C_{i}^{m}=0$ and $\chi_{i}^{m,(1)}$ as those associated with $C_{i}^{m}=1$. Then, (12) can be rewritten as

$$
\lambda_{i}^{m}=\ln \frac{\Sigma_{\beta \in \chi_{i}^{m,(1)}} P\left(\boldsymbol{s}_{i}=\beta \mid \hat{\boldsymbol{s}}_{i}\right)}{\Sigma_{\gamma \in \chi_{i}^{m,(0)}} P\left(\boldsymbol{s}_{i}=\gamma \mid \hat{\boldsymbol{s}}_{i}\right)}
$$

By assuming equiprobable $s_{i}$, using Bayes rule and applying the max-log-MAP approximation [17], we can approximate (13) by

$$
\lambda_{i}^{m} \approx \frac{1}{2 \sigma^{2}}\left[\min _{\beta \in \chi_{i}^{m,(0)}}\left\|\hat{\boldsymbol{s}}_{i}-K \gamma\right\|^{2}-\min _{\beta \in \chi_{i}^{m,(1)}}\left\|\hat{\boldsymbol{s}}_{i}-K \beta\right\|^{2}\right]
$$

where $\sigma^{2}$ is the variance of $n_{i}$.

It was shown in [17] that omitting the variance in (14) provides a slight improvement in performance at high SNR. In (14), $\lambda_{i}^{m}$ is expressed as the difference between two minimum squared Euclidean distances. The minimum squared Euclidean distance is simply a distance metric characterized by the $L_{2}$ norm in Euclidean space. In [17], this metric was generalized to an $L_{p}$-norm and it was found that decreasing $p$ to 1.9 provided some gain in performance over $p=2$. We extend this idea further to $p=1$ due to the simplicity of calculating the $L_{1}$-norm. Then, (14) takes the form

$$
\lambda_{i}^{m} \approx \min _{\gamma \in \chi_{i}^{m,(0)}}\left\|\hat{s}_{i}-K \gamma\right\|^{1}-\min _{\beta \in \chi_{i}^{m,(1)}}\left\|\hat{\boldsymbol{s}}_{i}-K \beta\right\|^{1}
$$

where $\|.\|^{1}$ denotes the $L_{1}$-norm. The calculation of the variance and the squaring operation are not used in (15), resulting in less computation than in (14). We have found that this yields good performance at low complexity. Note that including the noise variance at high SNR and using larger values of $p$ tends to magnify unreliable values of $\lambda_{i}^{m}$ and was shown in [17] to degrade performance. 


\section{Simulation Results}

All the simulation results presented are for MIMO systems with $^{6} n_{T}=n_{R}=4$. Gray mapped 16-QAM and QPSK constellations are used. We introduce the notation Modulation - QOSTBC - Detection to indicate the different modulations, QOSTBCs and detection schemes used in our BMCM systems. We use R1, R2 to denote the Rate 1 QOSTBC and Rate 2 QOSTBC, respectively. For example, the 16QAM-R2-PIC system uses 16-QAM, the Rate 2 QOSTBC, and PIC detection. For the uncoded systems, a frame is the length of the STBC block, $L$, while for coded systems, a frame has length $\left\lceil\frac{N}{R_{s t b c}}\right\rceil$ where $N$ is the block length of the LDPC component codes and $\lceil x\rceil$ denotes the smallest integer greater $\operatorname{than}^{7} x$. Each simulation point contains at least 100 frame errors. A frame is considered to be in error if and only if any of its data bits are in error.

In Section IV-A, results are presented for systems assuming the QS-Ind channel and perfect CSI at the receiver. We compare BMCM-QOSTBC-PIC performance to other published results at comparable throughputs. In Section IV-B, we examine the effects of imperfect channel estimation. In Section IV-D, we investigate the effects of removing the quasi-static fading assumption by comparing the performances in the QSfDT and NoQS-fDT channels, over a wide range of normalized fade rates.

\section{A. Perfect CSI}

Fig. 2 illustrates the coded bit error rate (BER) performance for the proposed 16QAM-R2-PIC system, using four $(343,186)$ LDPC component codes in a BMCM structure after $1,2,4,5$ and 9 PIC updates. The notation $B P=20(5)$ indicates that a maximum ${ }^{8}$ of $20 \mathrm{BP}$ iterations are used between PIC updates and 5 PIC updates are used in total. Thus, a maximum of $N_{\max }=120=20+5 \times 20$ BP iterations are used. The performance gain after more than 5 updates is minimal. The BER curve after 4 PIC updates is falling at almost 2 decades $/ d B$ and is not showing any signs of an error floor at $10^{-6}$. Each PIC update, coupled with BP decoding, provides diminishing improvement in performance.

Fig. 3 compares the uncoded BER performance of the Rate $1 / 2$ OSTBC of [6] using linear processing (OSTBC-LP) and the Rate 1 QOSTBC using JD [7]. In addition, we show the coded BER performance for the 16QAM-R1-JD, 16QAM-R1PIC and the QPSK-R2-PIC systems. The modulation schemes are all chosen to result in the same approximate throughput of 2 bits/symbol period. The uncoded Rate $1 / 2$ OSTBC has the worst performance in the group because it pays a penalty for the low value of $R_{s t b c}$, resulting in the required use of

\footnotetext{
${ }^{6}$ Although STBCs were originally proposed for one receive antenna, QOSTBCs introduce co-channel interference which increases the number of degrees of freedom required by the receiver to compensate the co-channel interference. For example, a Rate 2 QOSTBC symbol estimate contains 2 interference terms after linear processing, so at least 3 receive antennas are required to provide the necessary degrees of freedom. However, 4 receive antennas were chosen in order to provide a fair comparison to other systems.

${ }^{7}$ The LDPC component codes are zero-padded to ensure that the coded frame length is an integer.

${ }^{8}$ The BP algorithm uses a stopping criterion which will terminate BP decoding, between PIC updates, before 20 iterations if a codeword is found. PIC updates do not terminate early.
}

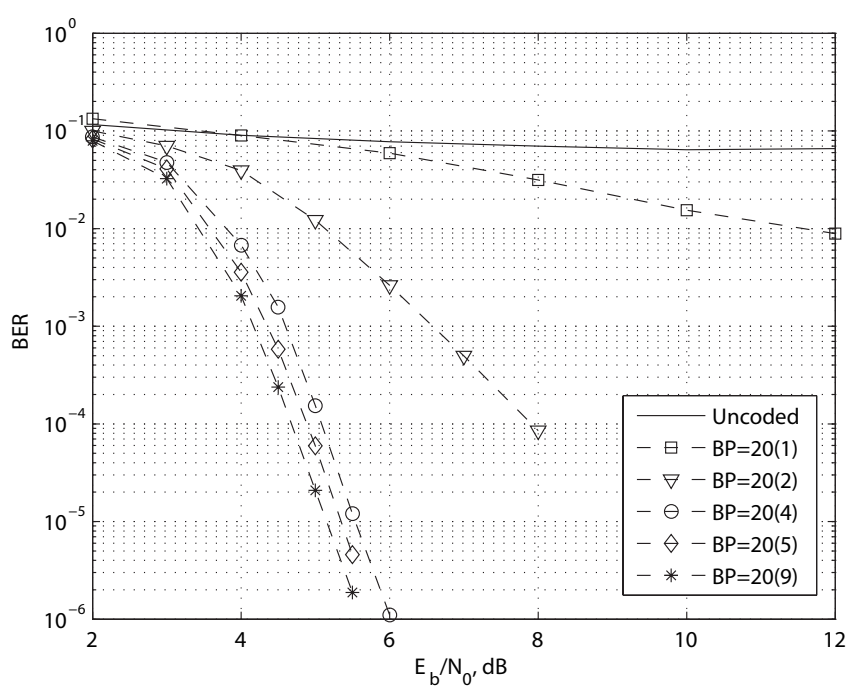

Fig. 2. BER performance of the proposed 16QAM-R2-PIC system using four $(343,186)$ LDPC component codes for 1, 2, 4, 5 and 9 PIC updates and a maximum of $20 \mathrm{BP}$ iterations between PIC updates. Throughput is 4.3 bits/symbol period.

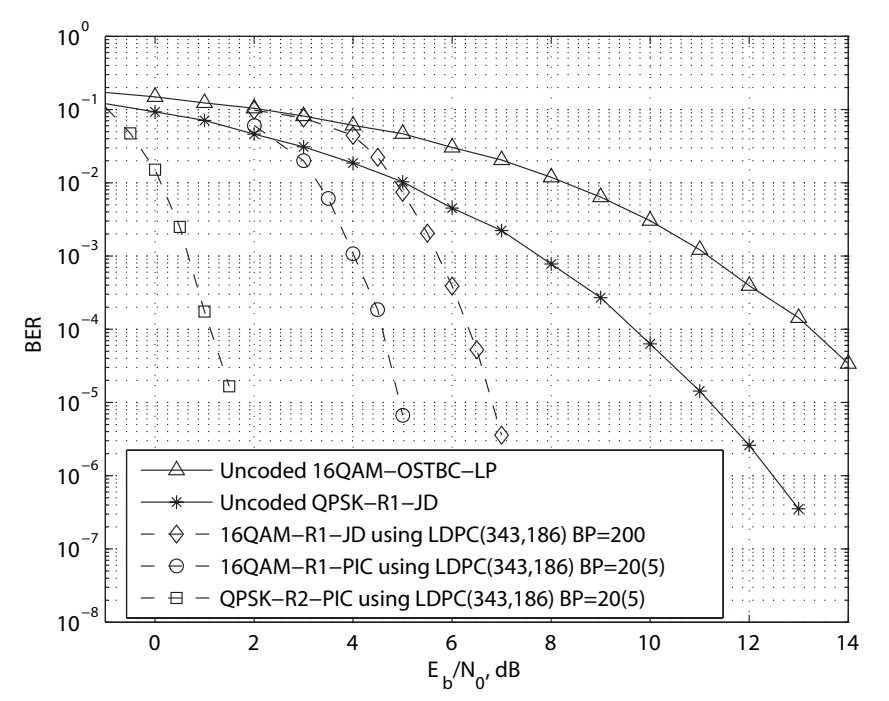

Fig. 3. BER performance comparison of coded and uncoded systems for the same approximate throughput of 2 bits/symbol period.

16-QAM and no LDPC codes. It attains a BER of $10^{-4}$ at 13.2dB. The Rate 1 QOSTBC using JD reaches the same BER at $9.7 d B$, a gain of $3.5 d B$ over the OSTBC. This is primarily because the Rate 1 QOSTBC has double the rate and so we can use a smaller constellation (QPSK) with a larger minimum Euclidean distance between constellation points.

We also compare the performance in Fig. 3 of the coded 16QAM-R1-JD and 16QAM-R1-PIC schemes using four $(343,186)$ LDPC component codes. When JD is used, the symbol estimates are demapped and the bit estimates are passed to the parallel BP decoders. A maximum of 200 BP iterations is used to decode each LDPC code. Due to the high complexity of JD, it is performed only once. When PIC is used, the symbol estimates on the first iteration are passed directly to the BP decoders after linear processing. On subsequent iterations, soft information from the BP decoders 


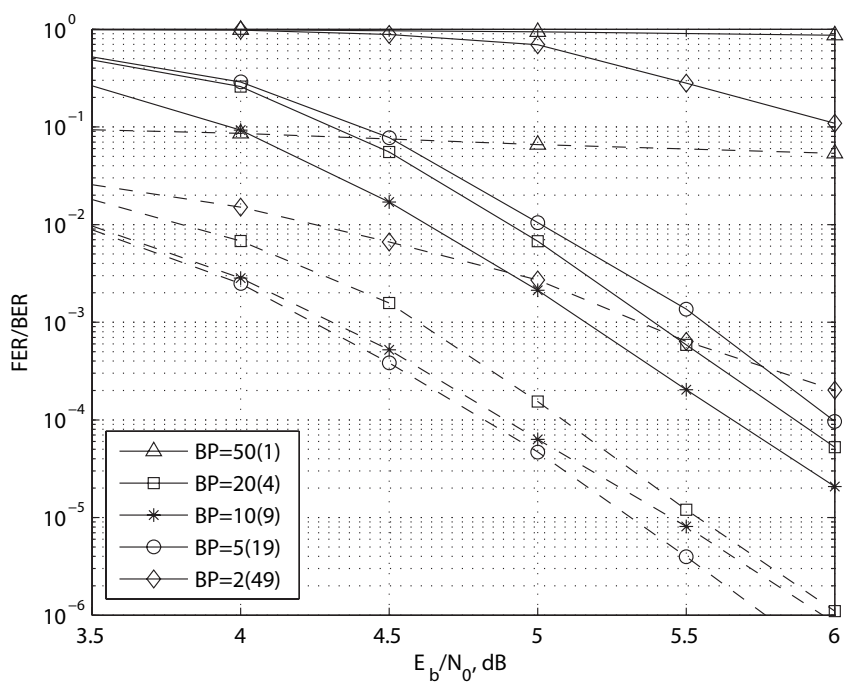

Fig. 4. FER (solid lines) and BER (dashed lines) performance of the 16QAM-R2-PIC BMCM system using $(343,186)$ LDPC component codes. Performance is shown for different numbers of PIC updates when an overall maximum of $N_{\max }=100 \mathrm{BP}$ iterations is used. Throughput is 4.3 bits/symbol period.

is passed to the PIC block. The coded 16QAM-R1-JD scheme achieves a BER of $10^{-4}$ at $6.4 d B$, a gain of $3.3 d B$ over the uncoded QPSK-R1-JD scheme. The coded 16QAM-R1-PIC scheme achieves the same BER at $4.6 d B$, a further gain of $1.8 d B$ over the coded 16QAM-R1-JD scheme.

When the Rate 2 QOSTBC is used with the same LDPC component codes and PIC, QPSK produces the required throughput. Fig. 3 shows that this achieves a BER of $10^{-4}$ at about $1.1 \mathrm{~dB}$, a gain of $3.5 \mathrm{~dB}$ over coded 16QAM-R1-PIC. This represents an overall gain of $12.1 d B$ over the uncoded Rate $1 / 2$ OSTBC.

Fig. 4 shows the frame error rate (FER) and BER performance of the 16QAM-R2-PIC scheme using $(343,186)$ LDPC component codes, providing a throughput of $4.3 \mathrm{bits} / \mathrm{symbol}$ period. Different numbers of PIC updates and BP iterations are used with $N_{\max }$ fixed at 100 . Performance generally improves as the number of PIC updates is increased. However, increasing the number of PIC updates beyond 9 and 19 degrades the FER and BER performances, respectively, because the number of allowable BP iterations between PIC updates decreases to a level such that the BP algorithm is unable to converge properly. To obtain good performance for a fixed $N_{\max }$, we have found that setting $\mathrm{BP}=\sqrt{N_{\max }}\left(\sqrt{N_{\max }}-1\right)$ provides a good starting point. This corresponds to a ratio of PIC updates to BP iterations (between updates) of approximately 1 . The effects on performance of changing this ratio are considered in detail in [18]. In Fig. 4, the best FER and the second best BER performances are produced by setting $\mathrm{BP}=10(9)$. This combination is used throughout the rest of this paper, unless otherwise stated.

We now compare the FER performance of the 16QAMR2-PIC BMCM system, the interleaved horizontal generalized layered space-time (HGLST) system ${ }^{9}$ of [10] and the LST-c

\footnotetext{
${ }^{9}$ This system was chosen due to its comparable throughput, hybrid spacetime code structure and values of $n_{T}$ and $n_{R}$.
}

layered space-time system ${ }^{10}$ of [13]. The interleaved HGLST scheme uses a 16-state space-time trellis code, 130 time slots in a frame and ordered SIC at the receiver. The LST-c scheme transmits QPSK symbols directly using interleaving over both space and time, instead of using space-time codes. It uses a $(504,252)$ LDPC component code on each transmit antenna, giving a frame length of 252 time slots. The LST-c scheme uses a modified PIC detector [15] with decision statistics combining to improve performance at the cost of increased complexity. Interleaving and deinterleaving are performed between the PIC detector and $n_{T} \mathrm{BP}$ decoders in an iterative fashion. In contrast, the BMCM-QOSTBC-PIC system employs a standard PIC detector, which is computationally less complex than either the modified PIC detector of [15] or ordered SIC. Constellation mapping and demapping are performed between the PIC detector with $M \mathrm{BP}$ decoders, and this significantly reduces the processing delay compared to interleaving and deinterleaving in [13]. We use four $(343,186)$ LDPC component codes and 172 time slots per frame.

All three systems employ $n_{T}=n_{R}=4$. The BMCM-QOSTBC-PIC (4.3 bits/symbol period) system outperforms the HGLST (4.0 bits/symbol period) and LST-c (4.0 bits/symbol period) systems by about $5.0 \mathrm{~dB}$ and $2.1 \mathrm{~dB}$, respectively, at FER $=10^{-2}$. The FER curve is falling at 2 decades $/ d B$, compared to only $0.5 \mathrm{decade} / d B$ for the other two. Therefore, we achieve greater diversity gain than these two systems.

\section{B. Imperfect CSI}

All the results presented thus far assume that perfect CSI is available at the receiver. Although this assumption is widely adopted, it is unrealistic. Here, we investigate the effects of imperfect channel estimation on BER performance, where the estimated CSI is modelled using (6). Fig. 5 shows the impact of CSI error on performance for the 16QAM-R1-JD and QPSK-R2-PIC systems. Both schemes use $(343,186)$ LDPC component codes. Compared to the perfect CSI case, the coded 16QAM-R1-JD system loses $1.2 \mathrm{~dB}$ and $3.9 \mathrm{~d} B$ at $\mathrm{BER}=10^{-4}$, for $\rho=0.98$ and $\rho=0.95$ respectively ${ }^{11}$. This is equivalent to estimation error energies of about $4 \%$ and $11 \%$ of the actual CSI, respectively. The coded QPSK-R2-PIC system loses only $0.5 d B$ and $1.2 d B$ in the same scenario. Simulations also found that the coded 16QAM-R1-PIC system loses $1.0 \mathrm{~dB}$ and $2.7 \mathrm{~dB}$ compared to perfect CSI. All these systems have a throughput of 2 bits/symbol period.

Higher order modulation schemes have a smaller minimum squared Euclidean distance between constellation points, and hence are more sensitive to channel estimation errors. Comparing the QPSK-R2-PIC and 16QAM-R1-PIC systems, we see that the SNR gain due to the extra estimation noise protection offered by QPSK exceeds the SNR loss due to the additional cochannel interference introduced by the Rate 2 QOSTBC. This demonstrates the effectiveness of using higher rate STBCs rather than higher order modulations to increase throughput.

\footnotetext{
${ }^{10}$ This system was chosen due to its comparable throughput, similar detection-decoding scheme and values of $n_{T}$ and $n_{R}$.

${ }^{11}$ Using fixed $\rho$ provides the worst-case performance. In [19], it was shown that $\rho$ approaches 1 with increasing SNR.
} 


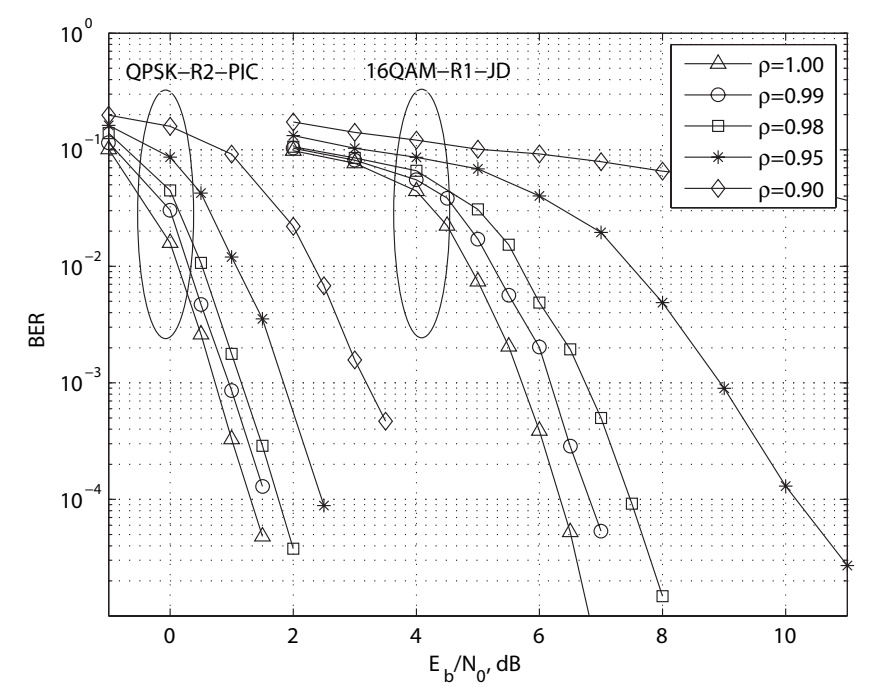

Fig. 5. BER performance for the 16QAM-R1-JD and QPSK-R2-PIC BMCM systems when $\rho=1,0.99,0.98,0.95$ and 0.90 . Both schemes use the $(343,186)$ LDPC component codes. Throughput is 2 bits/symbol period.
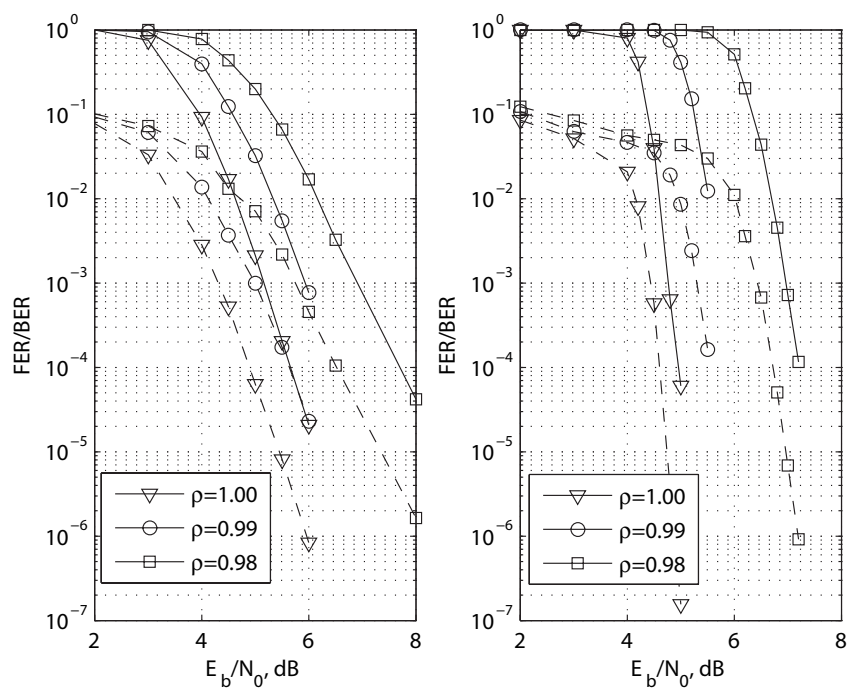

Fig. 6. FER (solid lines) and BER (dashed lines) performances for the 16QAM-R2-PIC system using (a) $(343,186)$ LDPC component codes (4.3 bits/symbol period) and (b) $(2197,1544)$ LDPC component codes $(5.6$ bits/symbol period). Perfect $(\rho=1)$ and imperfect $(\rho=0.99$ and 0.98$)$ CSI are considered. All LDPC codes use BP=10(9).

Comparing 16QAM-R1-JD and 16QAM-R1-PIC, we find that JD is more sensitive to imperfect CSI than PIC. The JD scheme also suffers a diversity loss in the presence of estimation errors. The iterative nature of PIC detection together with BP decoding improves robustness to channel estimation errors, compared to one-off JD detection followed by BP decoding.

It is obvious from Fig. 5 that the QPSK-R2-PIC system is very robust to channel estimation errors. It loses only about $2.5 d B$ compared to the perfect CSI case when $\rho=0.90$, which is equivalent to an estimation error energy of approximately $23 \%$. In addition, no diversity loss can be seen.

Fig. 6 show the effects of CSI estimation error on the FER and BER performances for 16QAM-R2-PIC using short $(343,186)$ and long $(2197,1544)$ LDPC component codes. Each frame corresponds to the length of the LDPC code,

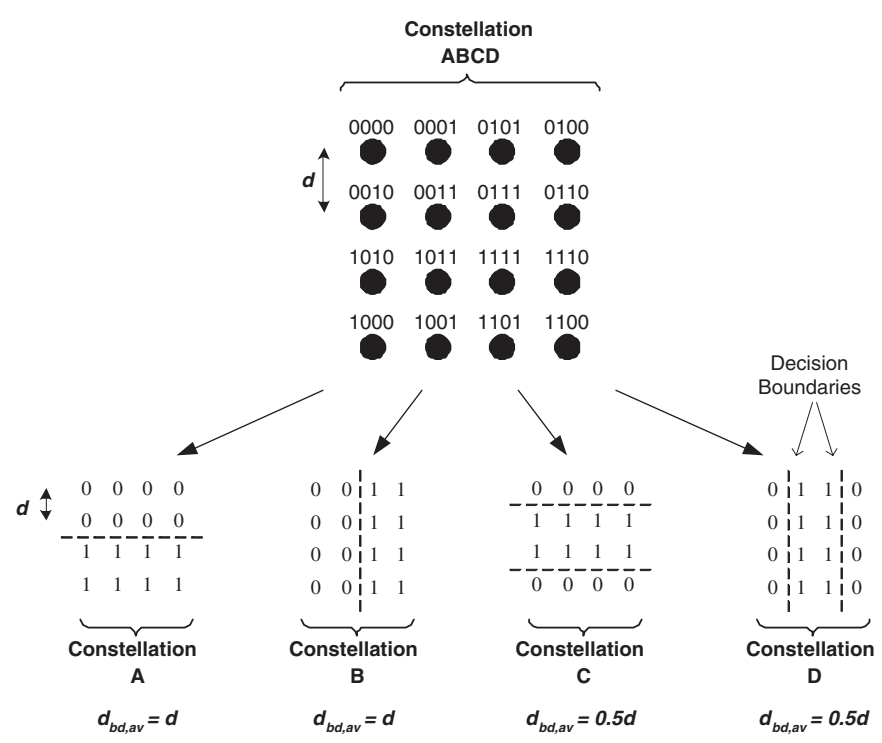

Fig. 7. Decision boundaries for each sub-label constellation of Gray-mapped 16-QAM.

which spans 172 and 1100 time slots, respectively, for the short and long LDPC codes. When $\rho=0.98$, the BER for the short LDPC codes reaches $10^{-4}$ at $6.5 d B$, an SNR loss of $1.6 d B$ from the perfect CSI case. When longer LDPC component codes are used, a loss of about $2.1 d B$ is observed. The long LDPC codes enable the system to achieve a throughput of 5.6 bits/symbol period. Both the FER and BER curves for the long LDPC codes are falling at 5 decades $/ d B$ (for $\rho=1$ ) and 4 decades/dB $(\rho=0.98)$. No error floors are observed down to $\mathrm{FER}=10^{-4}$ and $\mathrm{BER}=10^{-6}$. Throughput can be further increased by increasing $R_{l d p c}$, but this may lead to error floors.

\section{Performance with Unequal Error Correction}

In Gray-mapped 16-QAM systems, two of the four bits that label the constellation points are better protected than the other two, due to the average distance of each constellation point to the decision boundary corresponding to each bit [20]. This is illustrated in Fig. 7 where a Gray-mapped 16QAM constellation (denoted ABCD) is divided according to its four sub-labels (denoted $\mathrm{A}, \mathrm{B}, \mathrm{C}$ and $\mathrm{D}$, respectively). If $d$ is the minimum distance between any two constellation points, the average distance of each point to the closest decision boundary, $d_{b d, a v}$, is $d$ for sub-labels $\mathrm{A}$ and $\mathrm{B}$, and $0.5 d$ for sublabels $\mathrm{C}$ and $\mathrm{D}$. Therefore, sub-labels A,B are better protected against errors than sub-labels C,D.

Consider the 16QAM-R2-PIC system using $(2197,1663)$ LDPC component codes on all sub-labels, giving a system throughput of $6.1 \mathrm{bits} / \mathrm{symbol}$ period. We call this System I. Due to the unequal error protection between $A B$ and $C D$, our simulations show that the $\mathrm{AB}$ outperforms $\mathrm{CD}$ by about $1 d B$ at $\mathrm{BER}=10^{-5}$. We now introduce System II, which uses a $(2197,1728)$ LDPC code on AB and a $(2197,1596)$ LDPC code on $\mathrm{CD}$, giving the same system throughput of $6.1 \mathrm{bits} / \mathrm{symbol}$ period.

The advantage of using codes with unequal error correction capabilities on sub-labels with unequal error protection is 


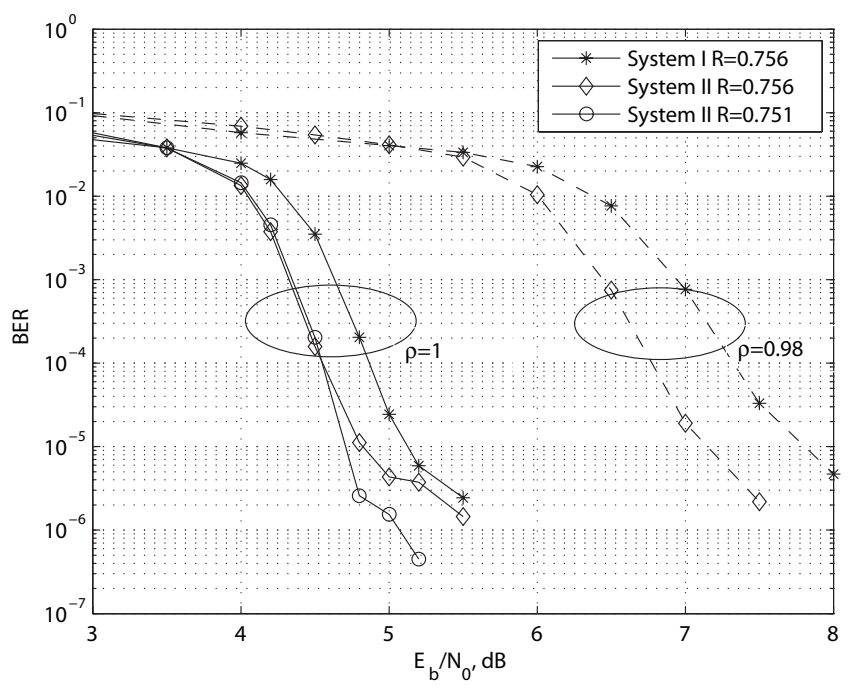

Fig. 8. BER performance comparison between 16QAM-R2-PIC systems. System I uses the same $(2197,1663)$ LDPC code on all sub-labels, giving $R_{l d p c}=0.756$. System II uses the $(2197,1728)$ LDPC code on $\mathrm{AB}$ and $(2197,1596)$ LDPC code on CD, giving $R_{l d p c}=0.756$. System III uses the $(2197,1702)$ LDPC code on AB and $(2197,1596)$ LDPC code on CD, giving $R_{l d p c}=0.751$. Performance is shown for both perfect $(\rho=1)$ and imperfect $(\rho=0.98)$ CSI

illustrated in Fig. 8. The BMCM structure allows each LDPC code to be matched to the different error protection of the corresponding sub-labels. By using a more powerful LDPC on the less protected sub-labels CD and a higher rate LDPC on the better protected sub-labels AB, System II achieves a $0.3 d B$ gain over System I at BER $=10^{-5}$, for the same throughput. In addition, it exhibits error floors at a slightly lower BER than System I and is more robust to channel estimation errors.

In Fig. 8, System III uses a more powerful but lower rate $(2197,1702)$ LDPC code on sub-labels AB, compared to the $(2197,1728)$ LDPC code used on sub-labels AB in System II. This decreases the system throughput by $0.8 \%$ to 6.0 bits/symbol period, but lowers the error floor. This illustrates the ability of the BMCM structure to provide a range of performance versus throughput tradeoffs.

\section{Fast and Slow Fading}

The quasi-static fading assumption in the QS-Ind and QSfDT channels allows detection using simple linear processing. However, this is not realistic in practice. Here, we investigate the performance of the BMCM-QOSTBC-PIC system in timevarying channels with a range of normalized fade rates, $f_{D} T$. Fig. 9 shows the effects of removing the quasi-static assumption, which transforms the QS-fDT channel into the NoQS-fDT channel, for $f_{D} T$ in the range of 0.1 to 0.0001 . We use QPSK and the Rate 2 QOSTBC with two $(343,186)$ LDPC component codes, giving a throughput of 2 bits/symbol period. A $1.6 d B$ loss is observed in very fast fading $\left(f_{D} T=0.1\right)$ at BER $=10^{-4}$. The SNR loss arises because the NoQS-fDT channel changes significantly over the $L$ time slots of the STBC when $f_{D} T=0.1$, contributing to large errors when the quasi-static assumption is used during linear processing. Removing the quasi-static assumption has almost no effect on

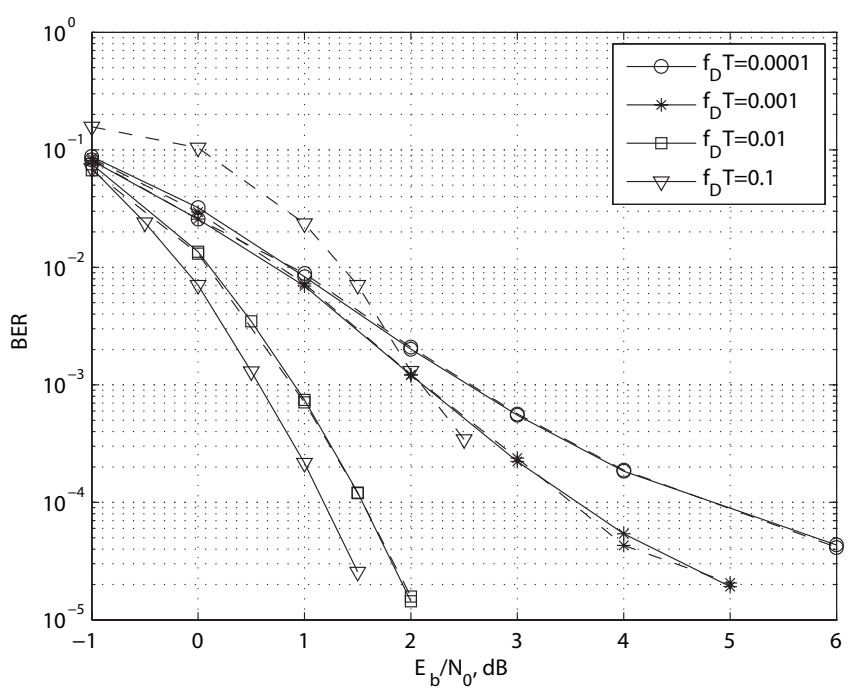

Fig. 9. BER performance for the QPSK BMCM Rate 2 QOSTBC using PIC in the QS-fDT channel (solid lines) and the NoQS-fDT channel (dashed lines), for normalized fade rates of $0.1-0.0001$. Both schemes use the $(343,186)$ LDPC component codes. Perfect CSI is assumed. Throughput is 2 bits/symbol period.

performance for the more realistic $f_{D} T$ values of 0.01 (fast fading) to 0.0001 (slow fading).

On the other hand, as $f_{D} T$ decreases from 0.01 to 0.0001 in both the QS-fDT and NoQS-fDT channels, the channel variation over a LDPC codeword decreases significantly. This results in a diversity loss as the fading becomes slower.

The simulation results of Fig. 9 for the QPSK-R2-PIC system use $\mathrm{BP}=10(9)$ in detection/decoding process. It was found that increasing the number of PIC updates (while maintaining $10 \mathrm{BP}$ iterations between updates) provides significant diversity and coding gains when the fading is slow $\left(f_{D} T<0.001\right)$. These gains are negligible in the fast fading scenario $\left(f_{D} T=0.01\right)$. In addition, increasing the number of $\mathrm{BP}$ iterations between the PIC updates provides no significant gains.

Although not shown here, the removal of the quasi-static assumption for the 16QAM-R2-PIC system results in an error floor at $\mathrm{BER}=10^{-2}$ when $f_{D} T=0.1$. When $f_{D} T=0.01$, the performance loss is about $0.1 \mathrm{~dB}$ at $\mathrm{BER}=10^{-4}$. For $f_{D} T$ in the range of $0.001-0.0001$, the loss is negligible. Removing the quasi-static assumption in the channel, but not in the detector, effectively results in CSI errors in fast fading. This is consistent with Fig. 5 where the 16QAM system is seen to be more sensitive to CSI errors than QPSK. As the fading slows, the change in CSI over adjacent time slots decreases and hence, the performance loss in going from the QS-fDT channel to the NoQS-fDT channel decreases.

Finally, we take another step towards more practical system assumptions by using the NoQS-fDT channel with a $2 \%$ ( $\rho=$ 0.99) channel estimation error in Fig. 10. We compare coded FER performance for the QPSK-R2-PIC ${ }^{12}$ and 16QAM-R1JD systems, using $(343,186)$ LDPC component codes. Here,

\footnotetext{
${ }^{12}$ The 16QAM-R2-PIC system is not used here because this requires a 256QAM-R1-JD system to obtain comparable throughputs. This requires JD to search through $2(256)^{2}=131,072$ possible pairs, for each block of 4 symbol estimates. Our aim is high throughput with feasible complexity.
} 


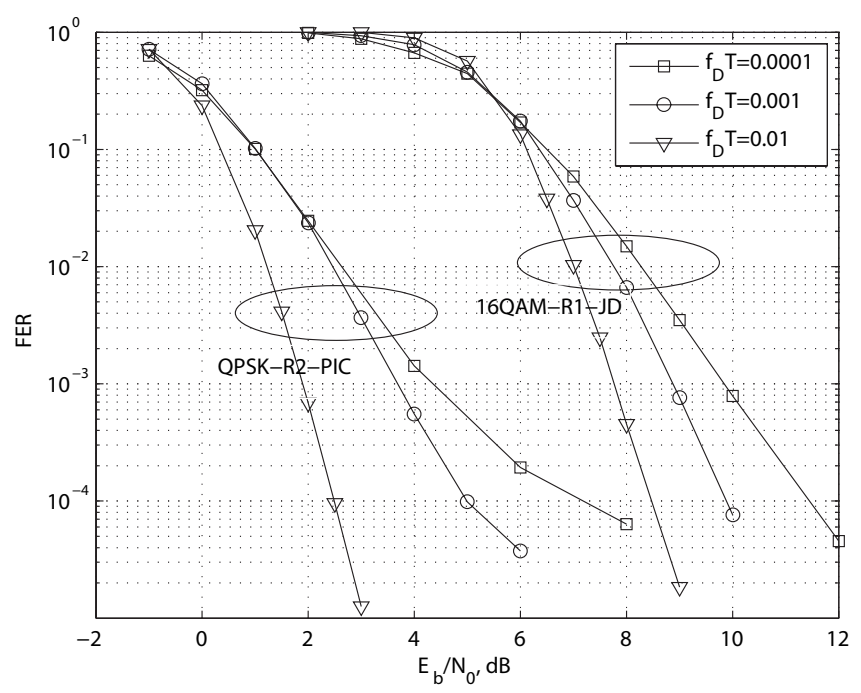

Fig. 10. FER performances for the QPSK-R2-PIC and 16QAM-R1-JD systems in the NoQS-fDT channel, for normalized fade rates of $0.01-0.0001$. Both systems use the $(343,186)$ LDPC component codes. CSI estimation error with $\rho=0.99$ is assumed. Throughput is 2 bits/symbol period.

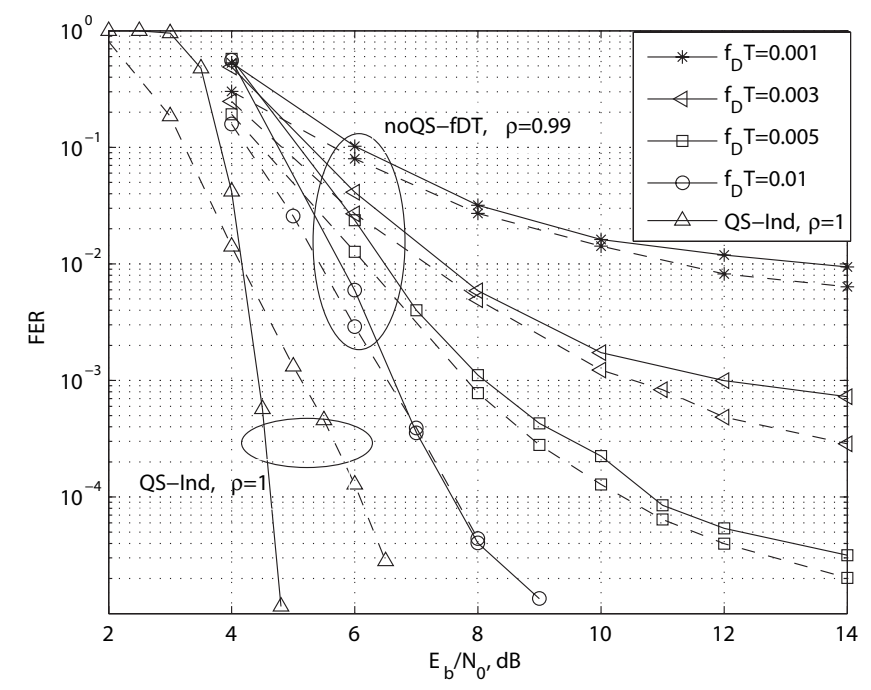

Fig. 11. FER performance comparison for 16QAM-R2-PIC systems using BICM (solid lines) and BMCM (dashed lines) in the QS-Ind channel with $\rho=1$ and in the NoQS-fDT channel with $\rho=0.99$, and $f_{D} T=0.01-0.001$. $\mathrm{BP}=10(9)$ is used for both systems. Throughput is $4.3 \mathrm{bits} / \mathrm{symbol}$ period.

we use BP=10(19) for the PIC system, giving $N_{\max }=200$. The JD system also uses $N_{\max }=200$.

When the fading is slow $\left(f_{D} T=0.0001\right)$, QPSK-R2-PIC outperforms 16QAM-R1-JD by about $5.5 d B$ at FER $=10^{-3}$. However, the QPSK-R2-PIC system suffers a diversity loss at high SNR. This loss can be eliminated by employing more than 19 PIC updates (and hence larger $N_{\max }$ ). In fast fading $\left(f_{D} T=0.01\right)$, QPSK-R2-PIC outperforms 16QAM-R1-JD at the same FER by approximately $6 d B$, with no sign of an error floor. Similar trends are exhibited in the BER case, for both systems.

In Section II-A we compared the structure of bit-interleaved coded modulation (BICM) to BMCM. In Fig. 11, we compare the performance for both systems using 16-QAM and the Rate 2 QOSTBC. The BMCM system uses two $(343,216)$ LDPC codes on sub-labels $\mathrm{AB}$ and two $(343,156)$ LDPC codes on sub-labels $C D$ to match the unequal error protection of 16QAM. This gives an overall throughput of approximately 4.3 bits/symbol period. It spans 172 time slots. The BICM system uses a single $(1331,730)$ LDPC code and spans 167 time slots. Both systems have approximately the same system throughput.

In the QS-Ind channel with perfect CSI, BMCM outperforms BICM down to FER $=1 \times 10^{-2}$ and $\mathrm{BER}^{13}=4 \times 10^{-5}$. BICM outperforms BMCM by $1.5 d B$ at FER $=10^{-4}$, due to the higher minimum distance of the longer LDPC code. However, BMCM achieves comparable FER performance to BICM in the more realistic NoQS-fDT channel, for normalized $f_{D} T$ values of $0.01-0.001$ and a $2 \%$ CSI estimation error. BMCM also achieves FER error floors approximately 1.6 to 3 times lower than BICM. The BER error floors for BMCM are approximately 2 to 5 times lower than BICM at high SNR. The error floors rise as $f_{D} T$ decreases. As with the QPSK case in Fig. 9, these floors can be lowered by using more PIC updates.

As discussed in Section II-A, the BMCM structure shortens processing delay by a factor of 4 compared to BICM. In addition, unlike BICM, it does not suffer delays due to interleaving/de-interleaving.

\section{Conclusion}

We have developed a MIMO system which uses a Rate 2 QOSTBC concatenated with a BMCM structure at the transmitter. The proposed structure uses LDPC component codes and requires no interleaving, which reduces the overall processing delays. Any FEC codes may be used as component codes. At the receiver, linear processing is used with a simple iterative PIC detection scheme. The PIC scheme has much lower complexity than either the JD scheme of [7] or the BLAST scheme of [8], [2], [10] (which employs ordered SIC). The LDPC codes allow for low complexity decoding using the $\mathrm{BP}$ algorithm. The flexibility of the BMCM structure provides for a range of performance versus throughput tradeoffs.

Simulation results show that we are able to achieve a throughput of 5.6 bits/symbol period using $n_{T}=n_{R}=4$ and 16-QAM and still maintain very good BER and FER performance, when perfect CSI is available and quasi-static fading is assumed. The use of a high rate QOSTBC enables the use of a smaller modulation scheme, for fixed throughput, thereby providing better overall noise protection. When the quasi-static assumption is removed, the proposed system maintains good performance in fast to slow fading $\left(f_{D} T=0.01-0.001\right)$. The results also show that the proposed system is robust to channel estimation errors, with the QPSK-R2-PIC scheme losing only $2.5 d B$ in the presence of a $23 \%$ channel estimation error, with no diversity loss.

While BICM benefits from the use of longer codewords in independent quasi-static channel with perfect CSI, BMCM is more robust when more realistic assumptions used. BMCM also has shorter processing delays. 


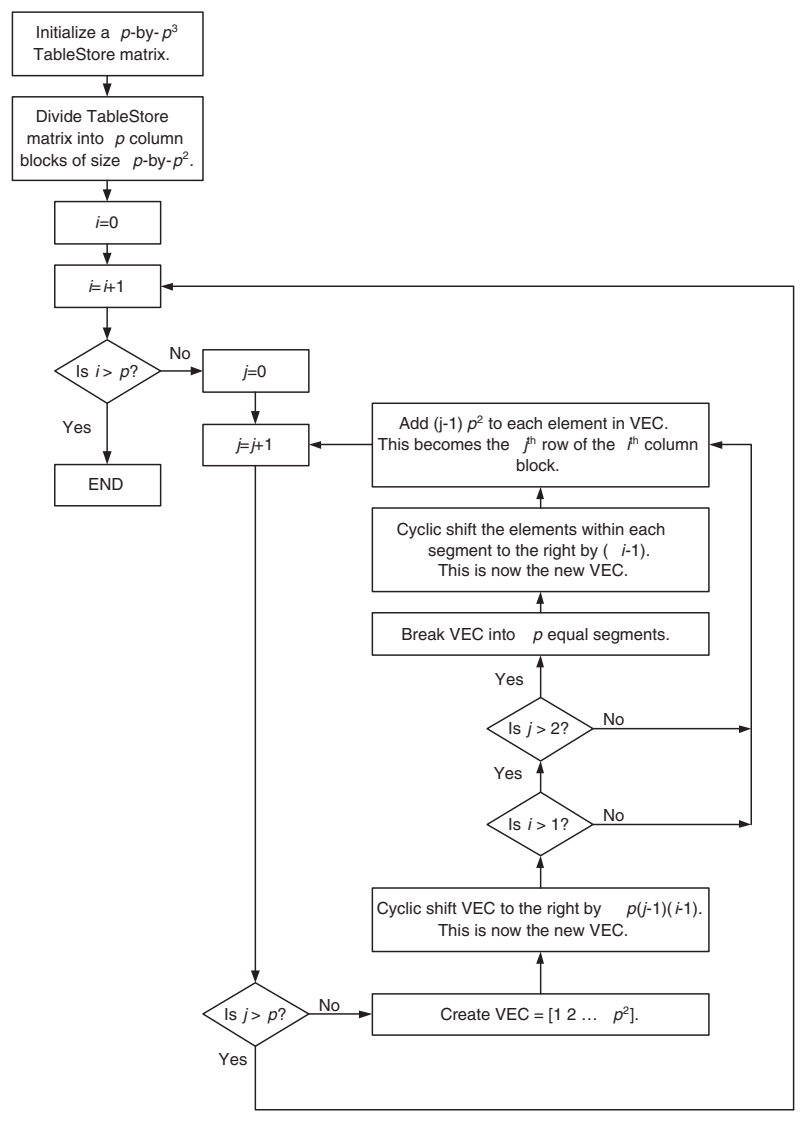

Fig. 12. Flow diagram to generate the TableStore matrix for the proposed LDPC code construction.

\section{APPENDIX}

\section{APPENDIX I - LDPC CODE CONSTRUCTION}

We outline our LDPC code construction method, which can create codes with different rates for the same block length. To the best of our knowledge, it is a novel method. It creates regular LDPC codes, which have parity check matrices, $\boldsymbol{H}$, with a constant row and column weight of $p$.

First, we create a $p$-by- $p^{3}$ matrix called TableStore. The algorithm used to generate TableStore is described in Fig. 12. For example, for $p=2$, the 2-by- 8 TableStore matrix generated is given by

$$
\left[\begin{array}{llllllll}
1 & 2 & 3 & 4 & 1 & 2 & 3 & 4 \\
5 & 6 & 7 & 8 & 7 & 8 & 5 & 6
\end{array}\right]
$$

The elements in the $k^{\text {th }}$ column of TableStore specify the rows of $\boldsymbol{H}$ containing a 1 in that column. The other rows have values of 0 . The TableStore matrix of (16) defines an 8-by-8 $\boldsymbol{H}$ matrix which has a constant row weight and column weight of $p=2$. Our construction algorithm generates a $p^{3}$ by- $p^{3}$ square matrix $\boldsymbol{H}$, which can be divided into $p$-by- $p$ identity matrices, each of which are permuted in such a way that the girth is at least 6 . By systematically removing blocks of $p$ rows of $\boldsymbol{H}$, we can construct a set of generator matrices with increasing values of $K$ and fixed values of $N=p^{3}$. Removing rows of $\boldsymbol{H}$ does not reduce the girth.

\footnotetext{
${ }^{13}$ The BER curves for BICM and BMCM are not shown due to space constraints.
}

\section{REFERENCES}

[1] A. Stefanov and T. Duman, "Turbo-coded modulation for systems with transmit and receive antenna diversity over block fading channels: System model, decoding approaches, and practical considerations," IEEE J. Select. Areas Commun., vol. 19, no. 5, pp. 958-969, May 2001.

[2] G. J. Foschini, "Layered space-time architecture for wireless communication is a fading environment when using multiple antennas," Bell Labs. Tech. J., vol. 1, no. 2, Autumn 1996.

[3] A. Ohhashi and T. Ohtsuki, "Performance analysis and code design of low-density parity check (LDPC) coded space-time transmit diversity (STTD) systems," in Proc. IEEE Globecom, vol. 5, no. 29, pp. 31183122, Nov.-Dec. 2004.

[4] D.-F. Yuan, P. Zhang, and Q. Wang, "The concatenation scheme MLCSTBC combining MLC and STBC over rayleigh fading channels," in Proc. MILCOM, vol. 2, no. 28-31, pp. 1300-1304, Oct. 2001.

[5] S. Alamouti, "A simple transmit diversity technique for wireless communications," IEEE J. Select. Areas Commun., vol. 16, no. 8, pp. 14511458, Oct. 1998.

[6] V. Tarokh, H. Jafarkhani, and A. R. Calderbank, "Space-time block codes from orthogonal designs," IEEE Trans. Inform. Theory, vol. 45, no. 5, pp. 1456-1467, July 1999.

[7] H. Jafarkhani, "A quasi-orthogonal space-time block code," IEEE Trans. Commun., vol. 49, no. 1, pp. 1-4, Jan. 2001.

[8] E. N. Onggosanusi, A. G. Dabak, and T. A. Schmidl, "High rate spacetime block coded scheme: performance and improvement in correlated fading channels," in Proc. WCNC, vol. 1, pp. 194-199, Mar. 2002.

[9] V. Tarokh, A. Naguib, N. Seshadri, and A. R. Calderbank, "Combined array processing and space-time coding," IEEE Trans. Inform. Theory, vol. 45, pp. 1121-1128, May 1999.

[10] M. Tao and R. S. Cheng, "Generalized layered space-time codes for high data rate wireless communications," IEEE Trans. Wireless Commun., vol. 3, no. 4, pp. 1067-1075, July 2004.

[11] E. Zehavi, "8-PSK trellis codes for a rayleigh channel," IEEE Trans. Commun., vol. 40, pp. 873-884, May 1992.

[12] B. Manatsavee, K. Ahmed, and A. Fernando, "Performance of PIC,SIC and decorrelating detectors for MUD technique in WCDMA system," in Proc. ICICS-PCM, vol. 2, pp. 892-896, Dec. 2003.

[13] K. L. Lo, S. Marinkovic, Z. Chen, and B. Vucetic, "Performance comparison of layered space time codes," Proc. IEEE, vol. 3, pp. 1382 1387, Apr.-May 2002.

[14] M. Sellathurai and S. Haykin, "A simplified diagonal BLAST architecture with iterative parallel-interference cancellation receivers," in Proc. ICC, vol. 10, pp. 3067-3071, June 2001.

[15] S. Marinkovic, B. Vucetic, N. Ishii, S. Yoshida, and A. Ushirokawa, "Space-time iterative and multistage receiver structures for CDMA mobile communication systems," IEEE J. Select. Areas Commun., vol. 19, pp. 1594-1604, Aug. 2001.

[16] P. Sinha, A. Sinha, and D. Basu, "A novel architecture of a reconfigurable parallel DSP processor," in Proc. IEEE-NEWCAS, pp. 7174, June 2005.

[17] Q. Li, P. Du, and G. Bi, "Generalized soft decision metric generation for MPSK/MQAM without noise variance knowledge," in Proc. PIMRC, pp. 1027-1030, Sept. 2003.

[18] N. Pau, D. P. Taylor, and P. A. Martin, "Rate 2 quasi-orthogonal space time block codes using parallel interference cancellation," in Proc. VTCSpring, Melbourne, Australia, May 2006.

[19] S. J. Grant and J. K. Cavers, "Multiuser channel estimation for detection of cochannel signals," IEEE Trans. Commun., vol. 49, no. 10, pp. 18451855 , Oct. 2001.

[20] L. Hanzo, W. Webb, and T. Keller, Single- and Multi-Carrier Quadrature Amplitude Modulation, 1st ed. John Wiley \& Sons, Inc., 2000.

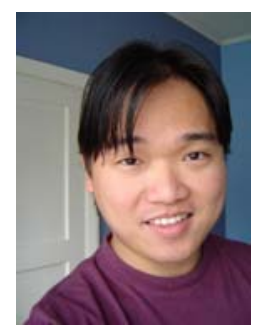

Nicholas Pau was born in Sibu, Sarawak, Malaysia on April 2, 1977. He received the B.Eng., M.Eng. and Ph.D. degrees from University of Canterbury, Christchurch, New Zealand in 1998, 2001 and 2007, respectively, in Electrical and Computer Engineering. In 2007, he was with the Communications Research Group at the department of Electrical and Computer Engineering of University of Canterbury from March to July. In Feb 2008, he started a lecturing contract there. His research interests include space-time codes, error-correcting codes, and iterative decoding/detection schemes. 


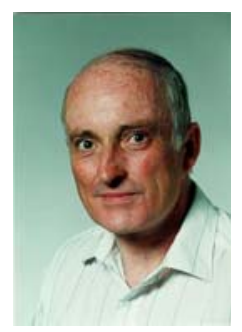

Desmond P. Taylor (M'63-F'94-LF'07) was born in Noranda, Quebec, Canada on July 5, 1941. He received the B.Sc.(Eng.) and M.Sc.(Eng.) degrees from Queen's University, Kingston, Ontario, Canada in 1963 and 1967 respectively, and the Ph.D. degree in 1972 in Electrical Engineering from McMaster University, Hamilton, Ontario, Canada. From July 1972 until June 1992, He was with the Communications Research Laboratory and Department of Electrical Engineering of McMaster University. In July 1992, he joined the University of Canterbury, Christchurch, New Zealand where he is the Tait Professor of Communications. His research interests are centered on digital wireless communications systems with a primary focus on the development of robust, bandwidth-efficient modulation and coding techniques, and the development of iterative algorithms for joint equalisation and decoding of the fading, dispersive channels typical of mobile radio communications. Secondary interests include problems in synchronisation, multiple access and networking. He is the author or co-author of approximately 220 published papers and holds two U.S. patents in spread spectrum communications. One paper won the S.O. Rice Award for the best Transactions paper in Communication Theory of 2001.

He is a Fellow of the IEEE, a Fellow of the Royal Society of New Zealand, and a Fellow of both the Engineering Institute of Canada and the Institute of Professional Engineers of New Zealand.

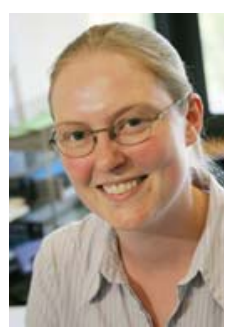

Philippa A. Martin (S'95-M'01-SM'06) received the B.E. (Hons. 1) and Ph.D. degrees in electrical and electronic engineering from the University of Canterbury, Christchurch, New Zealand, in 1997 and 2001, respectively.

From 2001 to 2004, she was a postdoctoral fellow, funded in part by the New Zealand Foundation for Research, Science and Technology (FRST), in the Department of Electrical and Computer Engineering at the University of Canterbury. In 2002, she spent 5 months as a visiting researcher in the Department of Electrical Engineering at the University of Hawaii at Manoa, Honolulu, Hawaii, U.S.A.. Since 2004 she has been working at the University of Canterbury as a lecturer and then as a senior lecturer (since 2007). In 2007, she was awarded the University of Canterbury, College of Engineering young researcher award. She served as an Editor for the IEEE Transactions on Wireless Communications 2005-2008 and regularly serves on technical program committees for IEEE conferences. Her current research interests include multilevel coding, error correction coding, iterative decoding and equalization, and space-time coding and detection, in particular for wireless communications. 